\title{
Mindfulness estado, habilidades mindfulness y auto-compasión en el aprendizaje de mindfulness:
}

\author{
Un estudio piloto
}

\author{
DANIEL CAMPOS BACAS \\ camposd@uji.es \\ AusiÀs CeBolla I Martí \\ acebolla@uji.es \\ PALOMA RAsal CANTÓ
}

\section{Resumen}

La investigación en mindfulness ha mostrado como esta habilidad, así como su entrenamiento, están relacionadas con bienestar psicológico. Paralelamente, han aparecido instrumentos para medir mindfulness sobre todo a nivel de rasgo. No obstante, mindfulness medido como estado no ha sido objeto de tanta investigación. El objetivo de este trabajo es estudiar la relación entre mindfulness estado (ME), mindfulness rasgo (MR), auto-compasión (AC) y las reacciones al aprendizaje de mindfulness. Una muestra de 18 profesionales participaron en un taller de formación en mindfulness, donde el eje central era la práctica a través de dos ejercicios esenciales: atención a la respiración (3 minutos) y el escáner corporal (body scan). Al inicio, los participantes contestaron los siguientes cuestionarios: escala ME (Mindfulness State Scale), cuestionario de MR (Five Facets of Mindfulness Questionnaire) y escala de auto-compasión. Después de cada ejercicio, respondieron la escala ME y el cuestionario de la experiencia mindfulness. Los resultados muestran como ME está relacionado con MR, así como con auto-compasión. Por otro lado, está relacionado con las reacciones a la práctica, y aumenta conforme avanza el aprendizaje. En conclusión, el estudio de ME y su medida puede aportar información muy útil sobre la experiencia de una persona en la práctica de mindfulness, así como en su aprendizaje.

Palabras clave: Mindfulness, Atención plena, Auto-compasión, Tratamientos psicológicos, Bienestar.

\section{Abstract}

Mindfulness research has shown how this skill as well as their training is related to psychological well-being. At the same time, tools to measure mindfulness have appeared, especially to measure trait level. However, mindfulness state (MS) measure has not received so much research. The objective of this study is to explore the relationship between MS, mindfulness trait (MT), self-compassion (SC) and reactions to 
mindfulness experience. A sample of 18 professionals participated in a mindfulness training workshop, where the training on mindfulness was implemented through two main exercises: mindfulness breathing exercise ( 3 minutes) and body scan exercise. Initially, participants completed the following questionnaires: State Mindfulness Scale (SMS), Five Facets of Mindfulness Questionnaire (FFMQ) and Self-Compassion Scale (scs). After each exercise, participants completed the SMS and the Mindfulness Experience Questionnaire. Results show how MS is related to MT, SC and mindfulness experience. Furthermore, MS is related to reactions to mindfulness practice and increases with learning. In conclusion, the study of mindfulness state and its measurement can provide useful information on the mindfulness experience, as well as their learning.

Keywords: Mindfulness, self-compassion, Psychological treatments, well-being.

\section{Introducción}

En los últimos años, en occidente se ha producido una asimilación del concepto mindfulness que ha derivado en un notable incremento de las terapias psicológicas que entrenan esta habilidad. Autores como Jon Kabat-Zinn, pionero en la aplicación de un programa de intervención basado en mindfulness, entienden mindfulness como la habilidad de «prestar atención de una manera determinada: de forma deliberada, en el momento presente y sin juzgar» (KabatZinn, 1994). En esta línea, Bishop et al., (2004) proponen un modelo de dos componentes: 1) la autorregulación de la atención de forma que se mantenga en la experiencia del momento presente; 2 ) la orientación deliberada de la atención hacia la propia experiencia con una actitud de curiosidad, apertura y aceptación de las sensaciones corporales, los pensamientos y las emociones.

Desde la tradición budista, mindfulness (Sati en Pali o Smrti en Sánscrito) se entiende como «presencia de mente, atención al presente» (Bodhi, 1993, p.86), concepto teórico y práctico que implica una «conciencia atenta del aquí-y-ahora» (Hollis-Walker y Colosimo, 2011). Bajo esta perspectiva, se define como «la habilidad mental de prestar atención a los eventos físicos o mentales que ocurren en el momento presente» (Bodhi, 1993). Siguiendo el Satipatthana Suta, un texto central para varias tradiciones budistas, se definen cuatro dominios u objetos de la experiencia a los cuales uno puede estar «mindful» (atento) como son el cuerpo, los sentimientos o las sensaciones, la conciencia y los objetos mentales.

En suma, podemos ver que mindfulness se entiende como «la capacidad humana universal y básica, que consiste en la posibilidad de ser conscientes de los contenidos de la mente momento a momento» (Simón, 2007). Por tanto, es una capacidad inherente al ser humano, que se puede entrenar, y puede ser potenciada mediante diferentes técnicas, siendo la meditación la más utlizada aunque no la única. Brown y Ryan (2004), afirman que «el beneficio principal de la práctica de la meditación es que cambia la conducta de los individuos en su vida diaria, aportando una presencia consciente no sólo respecto a sus eventos internos, sino también con su mundo cotidiano social y físico».

En la actualidad, la investigación en mindfulness ha experimentado un crecimiento casi exponencial de sus publicaciones (Black, 2009), mostrando cómo esta habilidad, así como su entrenamiento, están relacionados con bienestar psicológico (Brown y Ryan, 2003; Shapiro, Oman, Thoresen, Plante y Flinders, 2008). En este sentido, ha demostrado ser una técnica eficaz en el tratamiento de trastornos psicológicos (p.ej., ansiedad y depresión), así como en el 
tratamiento de enfermedades físicas o médicas (p.ej., Keng, Smoski y Robins, 2011; Khoury, et al., 2013). Además, en los últimos años la investigación en mindfulness ha aportado evidencias sobre cuáles son los mecanismos que subyacen a la eficacia (p.ej., Hözel et al., 2011; Shapiro et al. 2006). Aunque todavía no existen resultados concluyentes al respecto, en la literatura podemos encontrar una serie de iniciativas como la propuesta realizada por Hözel et al. (2011), que sugieren la regulación de la atención, la conciencia corporal, la regulación emocional y el cambio en la perspectiva del self, como mecanismos de mindfulness. En este sentido, otro constructo que ha mostrado estar implicado en mindfulness es la compasión, concretamente, la auto-compasión.

Desde la tradición budista, la compasión surge de forma natural con mindfulness; entendiendo la ubicuidad del sufrimiento y la profunda conexión que compartimos con los demás que sufren y el deseo de que, al igual que nosotros, estén bien (Hollis-Walker y Colosimo, 2011). El concepto de compasión hace referencia a la «experiencia sentida de compartir el dolor del otro, y el deseo de alivio de su sufrimiento» (Goldstein, 2003). Así, esta perspectiva quedaría lejos de la visión clásica occidental que entiende la compasión en términos de «pena» o «sentimiento de conmiseración y lástima que se tiene hacia quienes sufren penalidades o desgracias» (Real Academia Española, 2001, 22 ${ }^{\mathrm{a}}$ ed.).

Cuando la compasión se dirige hacia uno mismo se denomina auto-compasión y, según Neff (2003), conlleva amabilidad y entendimiento hacia uno mismo en términos de dolor o fracaso en lugar de ser auto-crítico con uno mismo; percibiendo las propias experiencias como parte de la experiencia humana en lugar de verlas como aislamiento; y observar los pensamientos y sentimientos dolorosos con mindfulness en lugar de sobre-identificarse con ellos. Por esta razón, una actitud compasiva hacia uno mismo puede decirse que implica una perspectiva mental equilibrada que se conoce como mindfulness (Neff, 2003).

En cuanto a la relación mindfulness y auto-compasión, Hollis-Walker y Colosimo (2011) encontraron que mindfulness correlacionaba con auto-compasión $(r=0,69)$, al mismo tiempo que estaba mediando la relación mindfulness-felicidad. Por otra parte, Sharon Salzberg (2011) propone tres componentes de la práctica, concretamente de la meditación, que incluyen: 1) atención focal o concentración, 2) Atención abierta o mindfulness per se, y 3) Bondad amorosa o compasión. En este sentido, Kuyken et al. (2010) concluyen que cultivar una actitud de compasión hacia uno mismo, así como hacia las propias dificultades, puede ser uno de los mecanismos subyacentes a las intervenciones basadas en mindfulness.

Podemos ver como algunos estudios empíricos, así como otros trabajos teóricos, empiezan a investigar la relación entre mindfulness y compasión o auto-compasión (p.ej., HollisWalker y Colosimo, 2011; Kuyken et al, 2010; Neff, 2003a). Sin embargo, todavía se requieren estudios que sigan explorando esta relación.

Paralelamente a estos hallazgos, han aparecido diferentes instrumentos para medir mindfulness, sobre todo a nivel de rasgo. La mayoría de estos estudios han mostrado la implicación e importancia de medir mindfulness como rasgo (p.ej., Baer, Smith, Hopkins, Krietemeyer, y Toney, 2006; Baer et al., 2008; Brown y Ryan, 2003; Farb, 2012). No obstante, mindfulness medido como estado no ha sido objeto de tanta investigación. Mientras que medir esta habilidad como rasgo (MR) se refiere a la tendencia y voluntad de ser conscientes y mantener la atención a lo que está sucediendo en el presente. Mindfulness estado (ME) tiene que ver con estar o ser conscientes en un determinado momento (el presente) con una actitud determinada (estar «mindful»). Por tanto, ME trata de medir la experiencia en primera persona sentida en la práctica, y tendría la capacidad de medir la variabilidad temporal en la experiencia de mindfulness, así como la evolución en su comprensión y aprendizaje.

A pesar de la importancia que supone la medición y el estudio de mindfulness como estado (ME), actualmente no se cuenta con ninguna medición que esté diseñada para reflejar 
mindfulness como un comportamiento mental, como un estado, dependiente del contexto, y variable (Bishop, 2004; Sauer et al., 2013). Tampoco existe apenas investigación respecto a la relación entre MR y ME, así como sobre la implicación que tiene el ME en el entrenamiento o práctica de mindfulness. En cuanto a la relación ME y auto-compasión, no se encuentran estudios que aporten evidencias en este sentido. Los pocos estudios existentes sobre la relación mindfulness y auto-compasión consideran mindfulness como rasgo.

Por estos motivos, el presente trabajo tiene como objetivo principal estudiar la relación entre ME, MR, auto-compasión (AC) y las reacciones al aprendizaje de mindfulness. Concretamente, nos proponemos tres objetivos específicos: a) Estudiar la relación entre ME, MR y AC, b) estudiar la relación entre ME y la experiencia mindfulness después de cada ejercicio y, c) estudiar las diferencias en ME a lo largo del aprendizaje.

\section{Método}

\section{Participantes}

Para este estudio se reclutó a una muestra de 18 participantes (13 mujeres y 5 hombres) de un taller de formación en mindfulness de 2 horas. La media de edad de los participantes fue de 28,06 (DT=2,94) con un rango de edad de 23 a 33 años. El 66,67 \% de los participantes no tenía experiencia en la práctica formal de meditación, frente al 33,33 \% que sí tenia experiencia. En cuanto al nivel de estudios, todos los participantes eran licenciados en psicología, 4 de los cuales poseían el título de doctor en psicología.

\section{Instrumentos}

La Escala de mindfulness estado (State Mindfulness Scale, SMS; Tanay y Bernstein, 2013). La SMS se compone de 21 ítems con una escala de respuesta de 1 (En absoluto) a 5 (muy bien) para indicar si las frases describen bien sus experiencias en los últimos 15 minutos. Está compuesta por dos factores: I) Mindfulness estado de la mente $(\alpha=0,90)$ (p.ej., «Me dí cuenta de pensamientos yendo y viniendo» o «exploré activamente mi experiencia en el momento presente»), y II) Mindfulness estado del cuerpo $(\alpha=0,95)$ (p.ej., «fui consciente de lo que pasaba dentro de mi» o «me dí cuenta de sensaciones físicas yendo y viniendo»). La escala de mindfulness estado presenta una consistencia interna excelente en la puntuación total $(\alpha=0,95)$.

El Cuestionario de las Cinco Facetas de Mindfulness (FFMQ; Baer et al, 2006; Cebolla et al., 2012). El FFMQ es un auto-informe de 39 ítems para medir mindfulness rasgo. Los ítems se contestan en una escala Likert que va desde 1 (nunca o muy raramente verdad) a 5 (muy a menudo o siempre verdad), referidos a 5 facetas o habilidades de mindfulness: 1) Observar: atender a los hechos, emociones y otras respuestas conductuales sin intentar rechazarlas cuando resultan dolorosas ni prolongarlas cuando son placenteras (p.ej., «Noto cuando mi estado de ánimo empieza a cambiar»); 2) Describir: mide la capacidad de describir hechos y respuestas personales con palabras, y lo integran ítems como «Tengo problemas para expresar las palabras correctas que expresen como me siento»; 3) Actuar dándose cuenta: mide la capacidad de centrar la mente y la atención en la actividad que se está llevando a cabo, sin dividir la atención entre lo que hacemos y lo que pensamos; «Cuando hago tareas como limpiar o conducir, tiendo a soñar despierto/a o pensar en otras cosas»); 4) Aceptar (o permitir) sin juicios y 5) No reaccionar a la experiencia interna. Las cinco facetas muestran una buena con- 
sistencia interna, tanto en la versión inglesa como en la validación al castellano, obtieniéndose en esta última un coeficiente alfa de 0,88 en la puntuación total del FFMQ.

La Escala de auto-compasión (Self-Compassion Scale, scs; Neff, 2003b; García-Campayo et al., 2014;), es una escala de 26 ítems para medir auto-compasión que se dividen en 6 subescalas: amabilidad hacia uno mismo, auto-juicio, humanidad compartida, aislamiento, mindfulness y sobre-identificación. La subescala amabilidad hacia uno mismo (5 ítems) incluye ítems como "soy amable conmigo mismo/a cuando estoy experimentando sufrimiento» o «trato de ser cariñoso/a conmigo mismo/a cuando siento malestar emocional. La subescala Auto-juicio (5 ítems) se compone de ítems como por ejemplo «cuando veo aspectos de mi mismo/a que no me gustan, me critico continuamente». La subescala Humanidad compartida (4 ítems) se refiere a ítems como «cuando me siento ineficaz de alguna manera, trato de recordarme a mí mismo/a que los sentimientos de ineficacia son compartidos por la mayoría de la gente». La subescala Aislamiento está compuesta por 4 ítems, tales como «cuando pienso en mis deficiencias, tiendo a sentirme separado y aislado del resto del mundo». La subescala Mindfulness ( 4 ítems) incluye afirmaciones como «Cuando me siento deprimido trato de observar mis sentimientos con curiosidad y apertura de mente». Por último, la subescala Sobreidentificación está compuesta por 4 ítems, p.ej., "Cuando algo me molesta me dejo llevar por mis sentimiento». La escala de auto-compasión compuesta por 26 ítems, en la versión original, presenta una consistencia interna excelente $(\alpha=0,92)$.

El Cuestionario de la experiencia mindfulness, es una medida auto-elaborada compuesta por 14 ítems que se contestan en una escala de 5 puntos ( $1=$ nada $-5=$ Totalmente). El objetivo de esta escala es evaluar la experiencia de la práctica de mindfulness a través de dos subescalas: grado de comprensión (4 ítems) y reacciones a la práctica (10 ítems). La subescala grado de comprensión explora si la persona ha entendido claramente qué es mindfulness, cómo entrenarlo, cómo se aplica en la práctica clínica, y si tiene suficientes conocimientos como para utilizarlo en su vida cotidiana. Por otra parte, la subescala reacciones a la práctica mide la experiencia, concretamente las reacciones, que ha sentido la persona durante la práctica de mindfulness, a través de 10 ítems que se refieren a: 1) Frustración, 2) Entender lo que tenía que hacer, 3) He podido concentrarme en la instrucción, 4) He sentido mareo, 5) Desear que el tiempo pasara rápido y abrir los ojos, 6) Distraído, 7) Relajado, 8) Somnolencia, 9) Ansiedad, y 10) Ver los pensamientos ir y venir sin quedarse atrapado/a por ellos.

\section{Procedimiento}

Antes del taller de formación todos los participantes contestaron la escala de ME, FFMQ y scs. Al inicio del taller, se realizó una breve introducción teórica al concepto de mindfulness, así como a su utilidad terapéutica, cuáles son las habilidades implicadas y cómo se introduce en terapia, para continuar con la práctica de mindfulness a través de dos ejercicios esenciales: atención a la respiración durante 3 minutos y el escáner corporal (body scan). En el ejercicio de atención a la respiración (3 minutos) los participantes seguían las instrucciones del profesor que les invitaban a dirigir y mantener su atención en la respiración, concentrándose en ésta, tal y como era, en el momento presente. En el ejercicio del escáner corporal, los participantes exploraban las sensaciones de su cuerpo, llevando la atención por todo el cuerpo, desde los pies a la cabeza, siguiendo las instrucciones del profesor. Después de cada ejercicio, los participantes respondieron de nuevo la escala de ME y el cuestionario de la experiencia de mindfulness. El taller tuvo una duración de 2 horas. 


\section{Resultados}

a) Relación entre ME, MR y AC

Para estudiar la relación entre ME, MR y auto-compasión (AC) se llevó a cabo un análisis de correlaciones de Pearson. Los resultados mostraron que no había correlaciones significativas entre ME con MR y AC antes de la intervención, aunque sí mostraron correlaciones significativas entre ME y la faceta Actuar dándose cuenta de MR ( $r=, 543 ; r=, 465)$, y entre la subescala de ME de eventos mentales, con la subescala sobre-identificación del cuestionario de autocompasión $(r=-, 483)$ tras la práctica del body scan.

\section{b) Relación entre ME y la experiencia mindfulness después de cada ejercicio}

En cuanto al grado de comprensión y las reacciones tras el primer ejercicio, los resultados (Tabla 1) mostraron correlaciones significativas entre ME (eventos mentales) con la sensación de estar distraído ( $r=-, 575)$, y con la comprensión del concepto de mindfulness $(r=, 512)$.

Tras la práctica del segundo ejercicio (body scan), la puntación entre ME (eventos mentales y cuerpo) correlacionó significativamente con la puntuación en los ítems concentración $(r=, 674 ; r=, 677)$ y con ver los pensamientos ir y venir sin quedarse atrapado/a por ellos $(r=, 594$; $r=, 589)$. La subescala de ME de eventos mentales también correlacionó significativamente con el ítem somnolencia $(r=-, 585)$.

Tabla 1

Correlaciones entre ME y la experiencia mindfulness después de cada ejercicio

\begin{tabular}{lcccc}
\hline \multicolumn{1}{c}{ EXPERIENCIA MINDFULNESS } & \multicolumn{2}{c}{ ME POST EJERCICIO 1 } & \multicolumn{2}{c}{ ME POST EJERCICIO 2 } \\
\hline & $\begin{array}{c}\text { EVENTOS } \\
\text { MENTALES }\end{array}$ & CUERPO & $\begin{array}{c}\text { EVENTOS } \\
\text { MENTALES }\end{array}$ & CUERPO \\
\hline Comprensión concepto mindfulness &, $512^{*}$ &, 337 &, 293 &, 173 \\
Entender cómo entrenar mindfulness &, 399 &, 224 &, 445 &, 401 \\
Entender la aplicación en la práctica &, 423 &, 172 &, 318 &, 210 \\
clínica &, 184 &, 271 &, 216 &, 170 \\
Tener suficientes conocimientos para & & & &,- 423 \\
utilizarlo en la vida cotidiana &, 097 &, 185 &,- 292 &,- 365 \\
Frustración &,- 319 &, 011 &,- 377 &, $677^{* *}$ \\
Entender lo que tenía que hacer &,- 148 &, 198 &, $674^{* *}$ &,- 261 \\
Concentración &, 431 &, 387 &,- 222 &,- 421 \\
Mareo &, 011 &, 272 &,- 450 &,- 400 \\
Deseo tiempo pasara rápido &,$- 575^{*}$ &,- 131 &,- 375 &, 016 \\
Distracción &, 135 &,- 020 &, 039 &,- 415 \\
Relajación &,- 138 &,- 118 &,$- 585^{* *}$ & \\
Somnolencia & & & &
\end{tabular}




\begin{tabular}{lcccc}
\hline \multicolumn{1}{c}{ EXPERIENCIA MINDFULNESS } & \multicolumn{2}{c}{ ME POST EJERCICIO 1 } & \multicolumn{2}{c}{ ME POST EJERCICIO 2 } \\
\hline & $\begin{array}{c}\text { EVENTOS } \\
\text { MENTALES }\end{array}$ & CUERPO & $\begin{array}{c}\text { EVENTOS } \\
\text { MENTALES }\end{array}$ & CUERPO \\
\hline Ansiedad &, 037 &,- 011 &,- 323 &,- 371 \\
Ver los pensamientos &, 434 &,- 329 &, $594^{* *}$ &, $589^{* *}$ \\
\hline
\end{tabular}

${ }^{* *} p<0,01 ;{ }^{*} p<0,05$

\section{c) Diferencias en ME a lo largo del aprendizaje}

Para estudiar si había diferencias en ME a lo largo del aprendizaje se realizó un ANOVA univariante de medidas repetidas. Como se muestra en la Figura 1, los resultados del ANOVA revelaron diferencias significativas en ambos factores de la escala de ME entre los diferentes momentos de evaluación (eventos mentales $F=5,456 ; p=, 009 ; \eta_{p}^{2}=, 234$; Cuerpo $F=3,449$; $\left.p=, 043 ; \eta_{p}^{2}=, 169\right)$. En general, se observa que ME aumenta a medida que avanza el aprendizaje de ME.

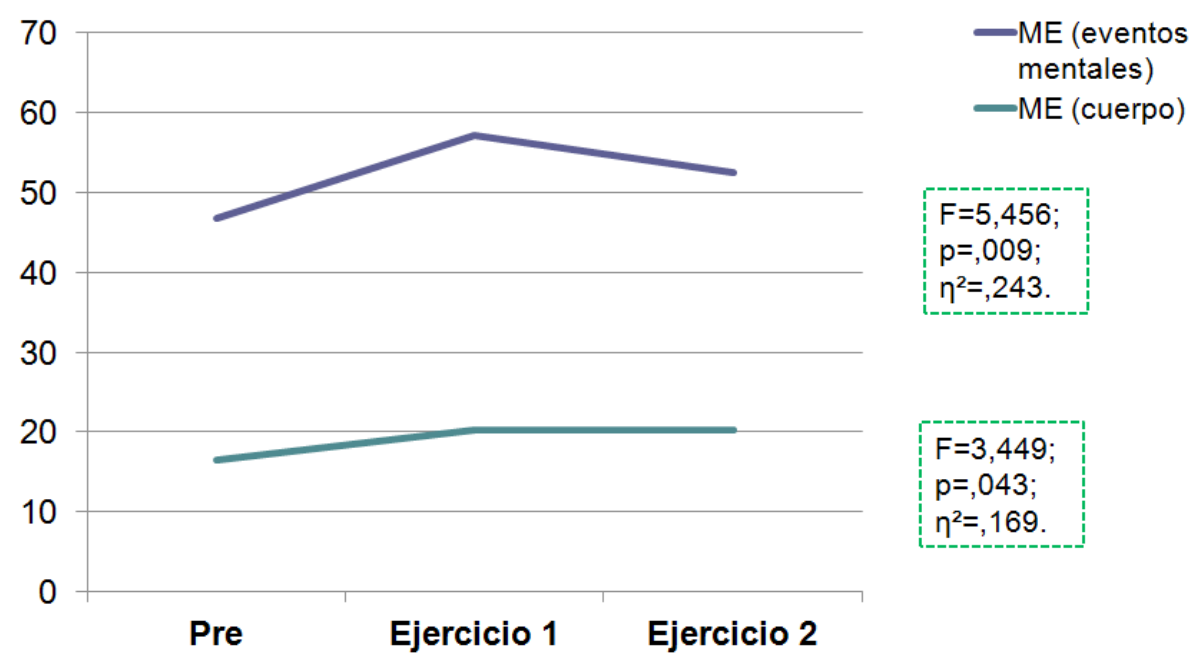

Figura 1. Puntuaciones en las subescalas de ME (eventos mentales y cuerpo): Antes del taller (pre), después del ejercicio 1 y después del ejercicio 2

\section{Discusión y conclusiones}

El objetivo de este trabajo era estudiar la relación entre ME, MR, auto-compasión (AC) y las reacciones al aprendizaje de mindfulness.

Los resultados indican que ME está relacionado con las habilidades mindfulness (O MR) y autocompasión, así como con las reacciones al aprendizaje. Además, ME aumenta a medida que avanza el aprendizaje.

Por otra parte, cabe destacar la relación entre la subescala eventos mentales de ME y la comprensión del concepto de mindfulness. Este dato presenta gran relevancia para la enseñanza de mindfulness, ya que parece indicar que la práctica favorece su comprensión. De 
esta forma, la experiencia meta-cognitiva de mindfulness, a través de la práctica, estaría facilitando el entendimiento y comprensión del concepto. Estos resultados van en la línea de otros estudios que sugieren la importancia de utilizar una metodología práctica y experiencial para el entrenamiento en habilidades y competencias de la técnica mindfulness (Cebolla y GarcíaPalacios, 2013).

No obstante, hay que tener en cuenta que este estudio presenta una serie de limitaciones al tratarse de un estudio piloto, como son: el bajo número de participantes, la poca duración del entretamiento (dos horas), no tener en cuenta la motivación de los participantes ante el aprendizaje de la técnica y, no tener en cuenta la experiencia en meditación de los participantes en los análisis estadísticos.

El presente trabajo pone de manifiesto la relevancia del estudio y medición de ME. Aunque la investigación en ME no ha sido muy extensa debido a la falta de instrumentos adecuados, su estudio puede aportar información muy útil sobre la experiencia de una persona en la práctica de mindfulness, así como en su aprendizaje. En un futuro, se requieren trabajos que sigan aportando evidencias en este sentido. Teniendo en cuenta el auge que ha experimentado el entrenamiento de mindfulness en las últimas décadas, parece necesaria la investigación sobre su enseñanza, así como acerca de los factores que pueden otimizarla y mediar en su comprensión.

\section{Referencias bibliográficas}

Baer, R. A., Smith, G. T., Hopkins, J., Krietemeyer, J. y Toney, L. (2006). Using self report assessment methods to explore facets of mindfulness. Assessment, 13, 27-45.

Baer, R. A., Smith, G. T., Lykins, E., Button, D., Krietemeyer, J., Sauer, S. ... Williams, J. M. G. (2008). Construct validity of the Five Facet Mindfulness Questionnaire in meditating and nonmeditating samples. Assessment, 15, 329-342.

Black, D. S. (2009). Hot Topics: A 40-year publishing history of mindfulness. Mindfulness Research Monthly, 1(5), 1.

Bodhi, B. (1993). A comprehensive manual of abhidhamma: The abhidhammattha sangaha of âcariya anuruddha. Seattle, WA: Pariyatti.

Brown, K. W., y Ryan, R. M. (2003). The benefits of being present: Mindfulness and its role in psychological well-being. Journal of Personality and Social Psychology, 84, 822-848.

Cebolla, A. y García-Palacios, A. (2013). Efectos de una aproximación experiencial en la adquisición de competencias asociadas al aprendizaje de la tècnica de mindfulness dentro de la asignatura de tecnicas de intervención y tratamientos psicologicos. En A. Bengochea (dir.), IV Jornada Nacional sobre Estudios Universitarios: El futuro de los títulos universitarios (pp.190-197). Castellón de la Plana, España: Publicacions de la Universitat Jaume I.

Cebolla, A., García-Palacios, A., Soler, J., Guillen, V., Baños, R., y Botella, C. (2012). Psychometric properties of the Spanish validation of the Five Facets of Mindfulness Questionnaire (FFMQ). Eur. J. Psychiat, 26(2), 118-126.

Farb, N. A (2012). Mind Your Expectations: Exploring the Roles of Suggestion and Intention in Mindfulness Training. The Journal of Mind-Body Regulation, 2 (1), 27-42.

Garcia-Campayo, J., Navarro-Gil, M., Andrés, E., Montero-Marin, J., López-Artal, L. y Demarzo, M. P. (2014). Validation of the Spanish versions of the long (26 items) and short (12 items) forms of the Self-Compassion Scale (SCS). Health and Quality of Life Outcomes, 12 (4).

Goldstein, J. (2003). Insight meditation. Boston and London: Shambhala. 
Hollis-Walker, L. y Colosimo, K. (2011). Mindfulness, self-compassion, and hapiness in non-meditators: A theoretical and empirical examination. Personality and Individual differences, 50, 222-227.

Hölzel, B. K., Lazar, S. W., Gard, T., Schuman-Olivier, Z., Vago, D. R., y Ott, U. (2011). How does mindfulness meditation work? Proposing mechanisms of action from a conceptual and neural perspective. Perspective in Psychological Science, 6, 537-559.

Kabat-Zinn, J. (1994). Wherever you go there you are: Mindfulness meditation in everyday life. New York, NY: Hyperion.

Keng, S.-L., Smoski, M. J. y Robins, C. J. (2011). Effects of mindfulness on psychological health: A review of empirical studies. Clinical Psychology Review, 31, 1041-1056.

Khoury, B., Lecomte, T., Fortin, G., Masse, M., Therien, P., Bouchard, V., Chapleau, M. A., Paquin, K. y Hofmann, S. G. (2013). Mindfulness-based therapy: A comprehensive meta-analysis. Clinical Psychology Review, 33, 763-771.

Kuyken, W., Watkins, E., Holden, E., White, K., Taylor, R. S., Byford, S., et al. (2010). How does mindfulness-based cognitive therapy work? Behaviour Research and Therapy, 48, 11051112.

Neff, K. D. (2003a). Self-compassion: An alternative conceptualization of a healthy attitude toward oneself. Self and ldentity, 2, 85-102.

Neff, K. D. (2003b). Development and validation of a scale to measure self compassion. Self and Identity, 2, 223-250.

Real Academia Española. (2001). Diccionario de la lengua española (22. ${ }^{a}$ ed.). Consultado en http://www.rae.es/rae.html

Sauer, S., Walach, H., Schmidt, S., Hinterberger, T., Lynch, S., Büssing, A., y Kohls, N. (2013). Assessment of mindfulness: Review on state of the art. Mindfulness, 4, 3-17.

Shapiro, S. L., Oman, D., Thoresen, C. E., Plante, T. G., y Flinders, T. (2008). Cultivating mindfulness: Effects on well-being. Journal of Clinical Psychology, 64, 840-862.

Shapiro, S. L.,. Carlson, L. E., Astin, J. A. y Freedman, B. (2006) Mechanisms of mindfulness. Journal of clinical psychology, 62(3), 373-386.

Simón, V. (2011). Aprender a practicar mindfulness. Barcelona: Sello editorial.

Tanay, G., y Bernstein, A. (2013, September 23). State Mindfulness Scale (SMS): Development and Initial Validation. Psychological Assessment. Advance online publication. 